\title{
Synthesis and Control of Flexible Systems with Component-Level Uncertainties
}

\author{
Peiman G. Maghami* \\ Senior Aerospace Engineer \\ Attitude Control Systems Engineering Branch \\ NASA Goddard Space Flight Center \\ Greenbelt, MD 20771 \\ Email: peiman.maghami@nasa.gov
}

\author{
Kyong B. Lim \\ Senior Research Engineer \\ Guidance and Control Branch \\ NASA Langley Research Center \\ Hampton, VA 23681
}

\begin{abstract}
An efficient and computationally robust method for synthesis of component dynamics is developed. The method defines the interface forces/moments as feasible vectors in transformed coordinates to ensure that connectivity requirements of the combined structure are met. The synthesized system is then defined in a transformed set of feasible coordinates. The simplicity of form is exploited to effectively deal with modeling parametric and non-parametric uncertainties at the substructure level. Uncertainty models of reasonable size and complexity are synthesized for the combined structure from those in the substructure models. In particular, we address frequency and damping uncertainties at the component level. The approach first considers the robustness of synthesized flexible systems. It is then extended to deal with non-synthesized dynamic models with component-level uncertainties by projecting uncertainties to the system level. A numerical example is given to demonstrate the feasibility of the proposed approach.
\end{abstract}

\section{Introduction}

Substructure synthesis is a well established area in modeling of flexible systems $[1-6]$. It is concerned with the modeling of the dynamics of substructures (or components) and then synthesizing them to predict the combined structural response. This synthesis is accomplished by enforcing deflection compatibility and force equilibrium at all substructure interfaces. There are several advantages of modeling via substructure synthesis; among these, (1) It allows much independence in the design and analysis of substructures, which is especially helpful if substructures are designed, fabricated, and even tested by different organizations. An example is the damping synthesis for the Space Shuttle [7] and more recently the component modules for the International Space Station, which are built by different companies in several countries. (2) It increases the power of existing finite-element analysis and design programs by allowing analysis/design by components especially in problems where too many finite element degrees of freedom are required to perform a dynamic analysis/design of the

\footnotetext{
* Address all correspondence to this author.

$\dagger$ 'This material is declared a work of the U.S. Government and is not subject to copyright protection in the United States. Approved for public release; distribution is unlimited.
} 
complete system. (3) It allows a direct synthesis of substructure test data. This is particularly useful for very large structural systems (such as the International Space Station) that cannot be tested as a whole. This also means that it can be used as a part of an experimental verification tool for substructures before deployment as a connected structure. (4) It directly connects model accuracy and refinement tests of components to overall performance and stability robustness predictions.

Active control of flexible systems based on component mode or substructure synthesis has received some attention in the recent past $[8-10]$. Since its earliest work in ref. [1], the primary goal of substructure synthesis methods has not changed much, i.e., to accurately predict the combined modal parameters: structural resonant frequencies and mode shapes. Consistent with the primary goal, most attention has been given to the issue of selecting a most effective subset of component (or assumed) modes and the related issue of modeling the substructure interfaces. Relatively little attention has been given to issues germane to active control, particularly in the area of robustness.

In applications of robust control to flexible systems, uncertainty models have been typically assumed for the combined (full-up) system. This approach is acceptable so long as these uncertainty models have been developed through experimental work. However, when ground testing of the assembled structure is not possible, uncertainty models can only be validated for the components. Consequently, methods are needed to provide for robust control design and analysis of flexible systems with the uncertainty defined at the component levels [9].

In this paper, we re-examine the substructure synthesis method from a controls perspective. In particular, our end goal is to develop a physically realistic and efficient way of modeling and controlling large flexible structures based on substructure (subsystems) models. As in ref. [9], we are ultimately concerned with the issues of closed loop stability and performance robustness due to inevitable modeling errors, configuration changes, or exogenous disturbances. In particular, we examine the issues of modeling uncertainties for the substructures and their influence on the response of the connected structure. The robustness of a synthesized system to uncertainties in the frequency and damping of its component modes is considered. The synthesized plant in transformed coordinates is placed in a Linear Fractional Transformation (LFT) form for robust design and analysis [11]. A formulation is also presented through which the uncertainties at the component level can be extended to system level uncertainties for design or analysis. This is quite useful in situations wherein while a global model is available, uncertainties are defined for the components only. Finally, the effects of component mode shape uncertainties as well as unmodeled component dynamics on the synthesized system are investigated and characterized in transformed coordinates. The proposed approach is applied to a two-dimensional flexible problem to demonstrate its feasibility.

\section{Substructure Synthesis}

For simplicity of presentation, consider two components, shown in Fig. 1, that have to be synthesized. These systems may represent two components of a larger flexible system (such as spacecraft or aircraft), or they may represent two selfsufficient systems that have joined for a specific purpose, such as two spacecraft docking. Consider the interface forces and moments (between the two components) as input forces yet to be determined. Moreover, assume that collocated and compatible with the interface forces/moments are linear/angular velocity outputs from each component. With these considerations, the linear and time-invariant dynamics of the two components are represented as follows

component 1

$$
\begin{gathered}
M_{1} \ddot{d}_{1}+D_{1} \dot{d}_{1}+K_{1} d_{1}=\tilde{B}_{1} u+\tilde{H}_{1} p_{1} \\
y_{1}=\tilde{C}_{r_{1}} \dot{d}_{1} \\
y_{p_{1}}=\tilde{L}_{d_{1}} d_{1}+\tilde{L}_{r_{1}} \dot{d}_{1}+\tilde{L}_{a_{1}} \ddot{d}_{1}
\end{gathered}
$$$$
\text { component } 2
$$

$$
M_{2} \ddot{d}_{2}+D_{2} \dot{d}_{2}+K_{2} d_{2}=-\tilde{B}_{2} u+\tilde{H}_{2} p_{2}
$$




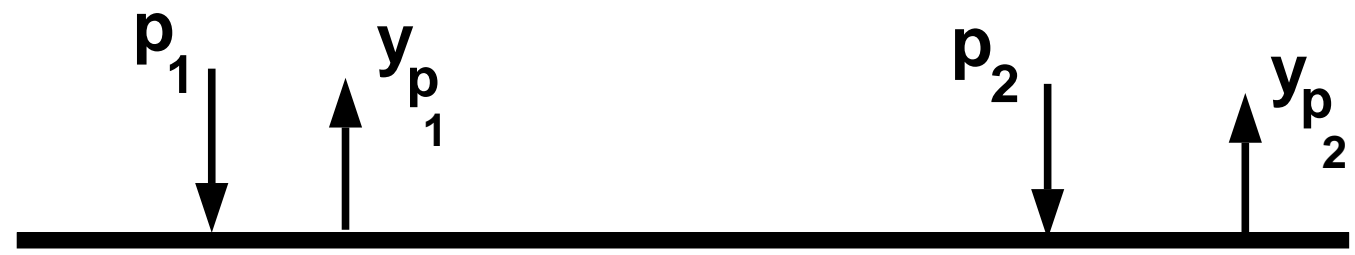

\section{Actual Structure}

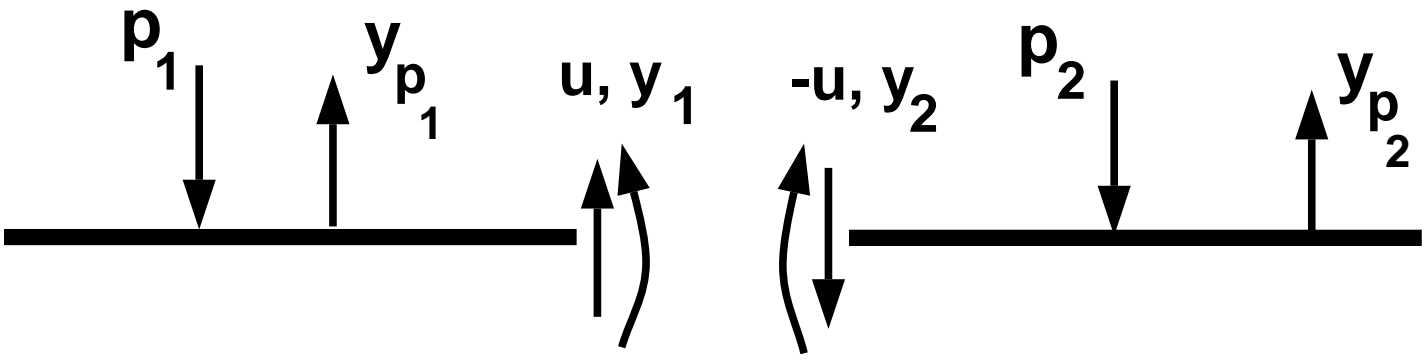

\section{Substructure Configuration}

Fig. 1. Synthesized substructures

$$
\begin{gathered}
y_{2}=\tilde{C_{r_{2}}} \dot{d}_{2} \\
y_{p_{2}}=\tilde{L}_{d_{2}} d_{2}+\tilde{L}_{r_{2}} \dot{d}_{2}+\tilde{L}_{a_{2}} \ddot{d}_{2}
\end{gathered}
$$

where, for component $i, d_{i}$ denotes the displacement vector; $M_{i}$ is the positive definite inertia matrix; $D_{i}$ is the damping matrix; $K_{i}$ is the non-negative definite stiffness matrix; $\tilde{B}_{i}$ is the influence matrix for the interface forces/moments; $u$ is the interface force/moment vector; $\tilde{H}_{i}$ is the influence matrix for the non-interface inputs; $p_{i}$ represents the non-interface inputs which include exogenous disturbances as well as control inputs for actual feedback control; $y_{i}$ is the interface velocity vector; $\tilde{C}_{r_{i}}$ is the corresponding output influence matrix; $y_{p_{i}}$ is the performance output vector; which could be a combination of displacement, velocity, and acceleration outputs; and $\tilde{L}_{d_{i}}, \tilde{L}_{r_{i}}$, and $\tilde{L}_{a_{i}}$ are the corresponding influence matrices. It is noted that the synthesis of the component dynamics may be achieved by only including velocity constraints at the interface. However, this may result in superfluous rigid-body modes in the synthesized dynamics. These modes can be avoided by including displacement constraints at the interface in addition to the velocity constraints. Since the order of a large flexible space structure (LFSS) can be quite large, for design and analysis purposes the order of the system is reduced to a design size using model reduction techniques such as modal truncation or modal cost analysis to obtain for component $\mathrm{i}, \mathrm{i}=1,2$

$$
\begin{gathered}
M_{r_{i}} \ddot{q}_{r_{i}}+D_{r_{i}} \dot{q}_{r_{i}}+K_{r_{i}} q_{r_{i}}= \pm \Phi_{i}{ }^{T} \tilde{B}_{i} u+\Phi_{i}{ }^{T} \tilde{H}_{i} p_{i} \\
y_{i}=\tilde{C}_{r_{i}} \Phi_{i} \dot{q}_{r_{i}} \\
y_{p_{i}}=\tilde{L}_{d_{i}} \Phi_{i} q_{r_{i}}+\tilde{L}_{r_{i}} \Phi_{i} \dot{q}_{r_{i}}+\tilde{L}_{a_{i}} \Phi_{i} \ddot{q}_{r_{i}}
\end{gathered}
$$




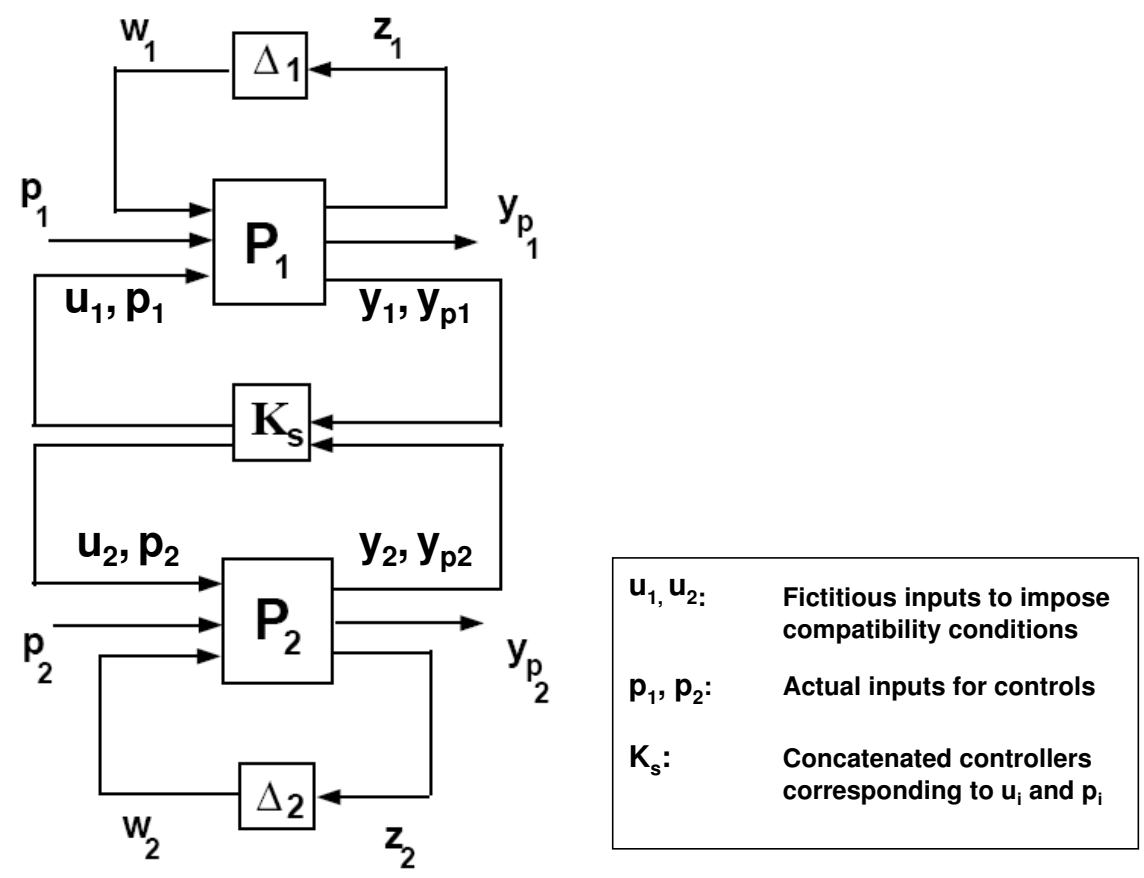

Fig. 2. Block Diagram of the Combined System

where $q_{r_{i}}$ is the vector of modal amplitudes obtained from transformation $d_{i}=\Phi_{i} q_{r_{i}} ; M_{r_{i}}, D_{r_{i}}$, $K_{r_{i}}$ are, respectively, the generalized inertia, damping and stiffness matrices; and $\Phi_{i}$ is a matrix whose columns are the $n_{i}$ open-loop eigenvectors associated with the included modes. If the mode shapes are normalized with respect to the inertia matrix, and modal damping is assumed, then $M_{r_{i}}=I_{n_{i} \times n_{i}}, D_{r_{i}}=\operatorname{Diag}\left\{2 \zeta_{1_{i}} \omega_{1_{i}}, \ldots, 2 \zeta_{n_{i i}} \omega_{n_{i i}}\right\}$, and $K_{r_{i}}=\operatorname{Diag}\left\{\omega_{1_{i}}^{2}, \ldots, \omega_{n_{i j}}^{2}\right\}$, where $\omega_{j_{i}}$ and $\zeta_{j_{i}}$, $j=$ $1, \ldots, n_{i}$ are the open-loop frequencies and damping ratios for the ith component. A block diagram for the combined system is given in Fig. 2. Here, each of the component plants are represented by P1 and P2, respectively. Each component model is assumed to have an LFT uncertainty structure representing its parametric and nonparametric uncertainties. The controller, $K_{s}$, includes the interfaces forces/moments that synthesize the combined system, as well as the actual feedback control signals for the combined system, which is assumed to be distributed among one or more components. For simplicity of presentation it is assumed that no physical (real) actuation is included in the interface between components. Defining the state vector $x_{i}=\left\{q_{r_{i}} \dot{q}_{r_{i}}\right\}^{T} ; i=1,2$, the second-order dynamics of the components can be rewritten in first-order forms as follows component 1

$$
\begin{gathered}
\dot{x}_{1}=A_{1} x_{1}+B_{1} u+H_{1} p_{1}+E_{1} w_{1} \\
y_{1}=C_{1} x_{1} \\
z_{1}=F_{1} x_{1}+\Gamma_{1} p_{1} \\
y_{p_{1}}=L_{1} x_{1}+D_{u_{1}} u+D_{p_{1}} p_{1}+D_{E_{1}} w_{1}
\end{gathered}
$$




$$
\begin{gathered}
\dot{x}_{2}=A_{2} x_{2}+B_{2} u+H_{2} p_{2}+E_{2} w_{2} \\
y_{2}=C_{2} x_{2} \\
z_{2}=F_{2} x_{2}+\Gamma_{2} p_{2} \\
y_{p_{2}}=L_{2} x_{2}+D_{u_{2}} u+D_{p_{2}} p_{2}+D_{E_{2}} w_{2}
\end{gathered}
$$

where $A_{i}, i=1,2$ denote the state matrices for each component. Matrices $B_{i}, H_{i}, E_{i}, C_{i}, F_{i}$, and $L_{i}, i=1,2$, represent the influence matrices for interface inputs, non-interface inputs, uncertainty outputs, interface outputs, uncertainty inputs, and non-interface output for each component, respectively. The matrices $D_{u_{1}}, D_{p_{1}}, D_{u_{2}}$, and $D_{p_{2}}$, represent feedthrough matrices associated with noninterface outputs of each component; $\Gamma_{1}, \Gamma_{2}, D_{E_{1}}$, and $D_{E_{2}}$ denote the feedthrough matrices associated with the uncertainty; $x_{1}$ and $x_{2}$ denote the state vectors of the components; $y_{1}$ and $y_{2}$ denote the interface velocity output vectors; $y_{p_{1}}$ and $y_{p_{2}}$ denote the noninterface output vectors' components which include performance outputs as well as measurement outputs for feedback control; $z_{1}$ and $z_{2}$ denote the uncertainty input vectors; $p_{1}$ and $p_{2}$ represent the noninterface inputs which include exogenous disturbances as well as control inputs for feedback control; and $w_{1}$ and $w_{2}$ denote the uncertainty output vector. The system matrices in the first-order forms are related to those in second-order forms as follows:

$$
\begin{gathered}
A_{i}=\left[\begin{array}{cc}
0 & I \\
-K_{r_{i}}-D_{r_{i}}
\end{array}\right] ; B_{i}=\left[\begin{array}{c}
0 \\
\pm \Phi_{i}{ }^{T} \tilde{B}_{i}
\end{array}\right] \\
H_{i}=\left[\begin{array}{c}
0 \\
\Phi_{i}^{T} \tilde{H}_{i}
\end{array}\right] ; C_{r_{i}}=\left[\begin{array}{ll}
0 \tilde{C}_{r_{i}} \Phi_{i}
\end{array}\right] ; \\
L_{i}=\left[\tilde{L}_{d_{i}} \Phi_{i}-\tilde{L}_{a_{i}} \Phi_{i} K_{r_{i}} \tilde{L}_{r_{i}} \Phi_{i}-\tilde{L}_{a_{i}} \Phi_{i} D_{r_{i}}\right] \\
D_{u_{i}}= \pm \tilde{L}_{a_{i}} \Phi_{i} \Phi_{i}^{T} \tilde{B}_{i} ; \quad D_{p_{i}}=\tilde{L}_{a_{i}} \Phi_{i} \Phi_{i}{ }^{T} \tilde{H}_{i}
\end{gathered}
$$

The influence matrices $E_{i}$ and $F_{i} ; i=1,2$, and the feedthrough matrices, corresponding to component uncertainties, will be discussed later.

For the two components to be connected, in a dynamical sense, one has to find an interface vector $u$, representing the internal forces and moments at the interface, such that the displacements and rotations of the two components at the interface remain identically the same. To achieve this, append the dynamics of the components, to obtain

$$
\left\{\begin{array}{l}
\dot{x}_{1} \\
\dot{x}_{2}
\end{array}\right\}=\left[\begin{array}{cc}
A_{1} & 0 \\
0 & A_{2}
\end{array}\right]\left\{\begin{array}{l}
x_{1} \\
x_{2}
\end{array}\right\}+\left[\begin{array}{l}
B_{1} \\
B_{2}
\end{array}\right] u+\left[\begin{array}{cc}
H_{1} & 0 \\
0 & H_{2}
\end{array}\right]\left\{\begin{array}{l}
p_{1} \\
p_{2}
\end{array}\right\}+\left[\begin{array}{cc}
E_{1} & 0 \\
0 & E_{2}
\end{array}\right]\left\{\begin{array}{l}
w_{1} \\
w_{2}
\end{array}\right\}
$$




$$
\begin{aligned}
& y \equiv y_{1}-y_{2}=\left[C_{1}-C_{2}\right]\left\{\begin{array}{l}
x_{1} \\
x_{2}
\end{array}\right\} \\
& z \equiv\left\{\begin{array}{l}
z_{1} \\
z_{2}
\end{array}\right\}=\left[\begin{array}{cc}
F_{1} & 0 \\
0 & F_{2}
\end{array}\right]\left\{\begin{array}{l}
x_{1} \\
x_{2}
\end{array}\right\}+\left[\begin{array}{cc}
\Gamma_{1} & 0 \\
0 & \Gamma_{2}
\end{array}\right]\left\{\begin{array}{l}
p_{1} \\
p_{2}
\end{array}\right\} \\
& y_{p} \equiv\left\{\begin{array}{l}
y_{p_{1}} \\
y_{p_{2}}
\end{array}\right\}=\left[\begin{array}{cc}
L_{1} & 0 \\
0 & L_{2}
\end{array}\right]\left\{\begin{array}{l}
x_{1} \\
x_{2}
\end{array}\right\}+\left[\begin{array}{l}
D_{u_{1}} \\
D_{u_{2}}
\end{array}\right] u+\left[\begin{array}{cc}
D_{p_{1}} & 0 \\
0 & D_{p_{2}}
\end{array}\right]\left\{\begin{array}{l}
p_{1} \\
p_{2}
\end{array}\right\}+\left[\begin{array}{cc}
D_{E_{1}} & 0 \\
0 & D_{E_{2}}
\end{array}\right]\left\{\begin{array}{l}
w_{1} \\
w_{2}
\end{array}\right\}
\end{aligned}
$$

The new output vector $y$ is the difference between the velocity of the two components at their interface. The problem is to find $u$ such that $y$ is identically zero for all $p_{1}$ and $p_{2}, w_{1}$ and $w_{2}$, and compatible $x_{1}(0)$ and $x_{2}(0)$. From Eq. (23), for $y$ to remain identically zero, $\left\{\begin{array}{l}x_{1} \\ x_{2}\end{array}\right\}$ must remain identically in the right null space of the matrix $\left[C_{1}-C_{2}\right]$. This means that an appropriate interface vector $u$ must be found that would render the system identically unobservable, i.e., $\left\{\begin{array}{l}x_{1} \\ x_{2}\end{array}\right\}$ identically remains in the undetectable subspace of the system given in Eqs. (22) and (23) for all $p_{1}$ and $p_{2}$, and $w_{1}$ and $w_{2}$. Let $N_{c}$ denote an orthonormal basis for the right null space of $\left[C_{1}-C_{2}\right]$, i.e.,

$$
\left[C_{1}-C_{2}\right] N_{c}=0
$$

and let $R_{c}$ denote an orthonormal complement to $N_{c}$. Transform the system in Eq. (22) via an orthogonal similarity transformation, such that

$$
\left\{\begin{array}{l}
x_{1} \\
x_{2}
\end{array}\right\}=\left[N_{c} R_{c}\right]\left\{\begin{array}{l}
\alpha \\
\beta
\end{array}\right\}
$$

From hereon, $\left\{\begin{array}{l}\alpha \\ \beta\end{array}\right\}$ will be referred to as the "transformed coordinates". The dynamics of the system in new coordinates becomes

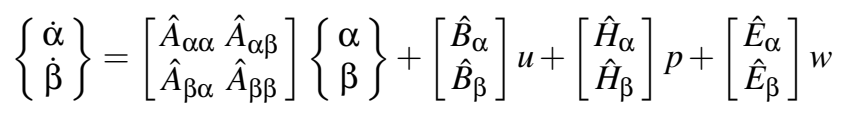

where $p=\left\{\begin{array}{l}p_{1} \\ p_{2}\end{array}\right\}, w=\left\{\begin{array}{l}w_{1} \\ w_{2}\end{array}\right\}$, and

$$
\begin{gathered}
{\left[\begin{array}{cc}
\hat{A}_{\alpha \alpha} & \hat{A}_{\alpha \beta} \\
\hat{A}_{\beta \alpha} & \hat{A}_{\beta \beta}
\end{array}\right]=\left[\begin{array}{ll}
N_{c} R_{c}
\end{array}\right]^{T}\left[\begin{array}{cc}
A_{1} & 0 \\
0 & A_{2}
\end{array}\right]\left[\begin{array}{ll}
N_{c} & R_{c}
\end{array}\right]} \\
{\left[\begin{array}{c}
\hat{B}_{\alpha} \\
\hat{B}_{\beta}
\end{array}\right]=\left[\begin{array}{ll}
N_{c} & R_{c}
\end{array}\right]^{T}\left[\begin{array}{l}
B_{1} \\
B_{2}
\end{array}\right]} \\
{\left[\begin{array}{c}
\hat{H}_{\alpha} \\
\hat{H}_{\beta}
\end{array}\right]=\left[\begin{array}{ll}
N_{c} & R_{c}
\end{array}\right]^{T}\left[\begin{array}{cc}
H_{1} & 0 \\
0 & H_{2}
\end{array}\right]}
\end{gathered}
$$




$$
\left[\begin{array}{l}
\hat{E}_{\alpha} \\
\hat{E}_{\beta}
\end{array}\right]=\left[\begin{array}{ll}
N_{c} & R_{c}
\end{array}\right]^{T}\left[\begin{array}{cc}
E_{1} & 0 \\
0 & E_{2}
\end{array}\right]
$$

Note that the linear and angular velocities at the interface are collocated and compatible with the forces and moments, i.e., $C_{1}=B_{1}^{T}$ and $-C_{2}=B_{2}^{T}$, such that along with Eq. (26), one has

$$
N_{c}^{T}\left[\begin{array}{l}
B_{1} \\
B_{2}
\end{array}\right]=N_{c}^{T}\left[C_{1}-C_{2}\right]^{T}=0
$$

resulting in

$$
\hat{B}_{\alpha}=N_{c}^{T}\left[\begin{array}{l}
B_{1} \\
B_{2}
\end{array}\right]=0
$$

From Eq. (27), $\left\{\begin{array}{l}x_{1} \\ x_{2}\end{array}\right\} \varepsilon \operatorname{Im}\left(N_{c}\right)$ iff $\beta(t)=0 ; \forall t$. The vector $u$ must be chosen such that $\beta(t)$, which represents the coordinates incompatible with the connectivity of the two components, remains identically zero for all $t, p, w$, with "compatible" initial conditions, i.e., $\beta(0)=0$. To accomplish this, the input vector $u$ must be chosen to render $\beta$ unreachable from $p$ and $w$, that is

$$
u=-\hat{B}_{\beta}^{-1}\left[\hat{A}_{\beta \alpha} \alpha+\hat{H}_{\beta} p+\hat{E}_{\beta} w\right]
$$

Although, any $u$ in the form of $u=-\hat{B}_{\beta}^{-1}\left[\hat{A}_{\beta \alpha} \alpha+\hat{H}_{\beta} p+\hat{E}_{\beta} w\right]+R \beta$, with $R$ as a non-destabilizing arbitrary matrix, would also be feasible, the condition for synthesizing the dynamics of the combined system necessitates that $\beta=0$ for all $t$, thereby reducing the expression for the interface forces and torques to that given in Eq. (35). It should be noted that the input (or controller) provided in Eq. (35) is not a concrete input (controller), but rather it is just a constraint to ensure compatibility of the components at their interface, which must be satisfied, no matter if uncertainties are present or not. The matrix inversion in Eq. (35) is guaranteed, as long as both influence matrices $B_{1}$ and $B_{2}$ are full rank, which they are since the interface forces are distinct. Therefore, since $B_{1}$ and $B_{2}$ are full rank, so is $\left[\begin{array}{l}B_{1} \\ B_{2}\end{array}\right]$, and so is $\hat{B}_{\beta}$, since $\hat{B}_{\beta}=R_{C}^{T}\left[\begin{array}{l}B_{1} \\ B_{2}\end{array}\right]$, and $R_{c}$ is an orthonormal basis for the column space of $\left[\begin{array}{l}B_{1} \\ B_{2}\end{array}\right]$. Using the vector $u$ of Eq. (35) in Eq. (28), with the aid of Eq. (34) simplifies to

$$
\left\{\begin{array}{c}
\dot{\alpha} \\
\dot{\beta}
\end{array}\right\}=\left[\begin{array}{cc}
\hat{A}_{\alpha \alpha} & \hat{A}_{\alpha \beta} \\
0 & \hat{A}_{\beta \beta}
\end{array}\right]\left\{\begin{array}{l}
\alpha \\
\beta
\end{array}\right\}+\left[\begin{array}{c}
\hat{H}_{\alpha} \\
0
\end{array}\right] p+\left[\begin{array}{c}
\hat{E}_{\alpha} \\
0
\end{array}\right] w
$$

It is obvious from this equation that since the initial conditions must be compatible for a combined system, such that $\beta(0)=0$, then $\beta$ remains identically zero for all $p, w$, and $t$, which means that, in the physical coordinates, $y_{1}=y_{2}$ for all $p$, $w$, and $t$. Therefore, compatibility conditions between the two components would be satisfied for all $p$ and $t$. The synthesized dynamics of the combined system can be written in a reduced-order form in the transformed coordinates, or in the original component-based coordinates. In the transformed coordinates, these dynamics are given as follows

$$
\begin{aligned}
& \dot{\alpha}=\hat{A}_{\alpha \alpha} \alpha+\hat{H}_{\alpha} p+\hat{E}_{\alpha} w \\
& z \equiv\left\{\begin{array}{l}
z_{1} \\
z_{2}
\end{array}\right\}=\hat{F}_{\alpha} \alpha+\hat{\Gamma}_{\alpha} p
\end{aligned}
$$




$$
y_{p} \equiv\left\{\begin{array}{l}
y_{p_{1}} \\
y_{p_{2}}
\end{array}\right\}=\hat{L}_{\alpha} \alpha+\hat{D}_{p_{\alpha}} p+\hat{D}_{E_{\alpha}} w
$$

where

$$
\begin{gathered}
\hat{F}_{\alpha}=\left[\begin{array}{cc}
F_{1} & 0 \\
0 & F_{2}
\end{array}\right] N_{c} \\
\hat{\Gamma}_{\alpha}=\left[\begin{array}{cc}
\Gamma_{1} & 0 \\
0 & \Gamma_{2}
\end{array}\right] \\
\hat{L}_{\alpha}=\left[\begin{array}{cc}
L_{1} & 0 \\
0 & L_{2}
\end{array}\right] N_{c} \\
\hat{D}_{p_{\alpha}}=\left[\begin{array}{cc}
D_{p_{1}} & 0 \\
0 & D_{p_{2}}
\end{array}\right] \\
\hat{D}_{E_{\alpha}}=\left[\begin{array}{cc}
D_{E_{1}} & 0 \\
0 & D_{E_{2}}
\end{array}\right]
\end{gathered}
$$

The dynamics of the combined system may also be presented in the original coordinates by writing the interface inputs (Eq. (35)) in terms of the original coordinates, and then using them in Eqs. (22)-(25).

\section{Component Model Uncertainty}

In this section, issues of parametric and nonparametric uncertainties in the component models and the way they affect the synthesized dynamics of the system will be considered. Parametric uncertainties include uncertainties in modal frequencies, damping ratios, and mode shapes of the components. The nonparametric uncertainties considered here are the unmodeled dynamics of the components. If modal models are used to represent the component dynamics, nonparametric uncertainties will include the truncated modes of the components. The development in this section uses the representation of the synthesized dynamics of the system in the transformed coordinates, as given in Eq. (37). This would make the treatment of parametric and nonparametric uncertainties easier. In this paper, parametric uncertainties in the component models in the form of frequency and damping uncertainties are fully treated. Moreover, the effects of parametric uncertainties in the component mode shapes as well as nonparametric uncertainties, in the form of unmodeled component dynamics, are discussed.

\subsection{Uncertainty in Frequency and Damping}

The synthesized dynamics of the combined system is illustrated in Fig. 3. Here, the plant $P$, representing the system dynamics, is given as

$$
\left\{\begin{array}{c}
\dot{\alpha} \\
y_{p} \\
z
\end{array}\right\}=\left[\begin{array}{ccc}
\hat{A}_{\alpha \alpha} & \hat{H}_{\alpha} & \hat{E}_{\alpha} \\
\hat{L}_{\alpha} & \hat{D}_{p_{\alpha}} & \hat{D}_{E_{\alpha}} \\
\hat{F}_{\alpha} & 0 & 0
\end{array}\right]\left\{\begin{array}{l}
\alpha \\
p \\
w
\end{array}\right\}
$$




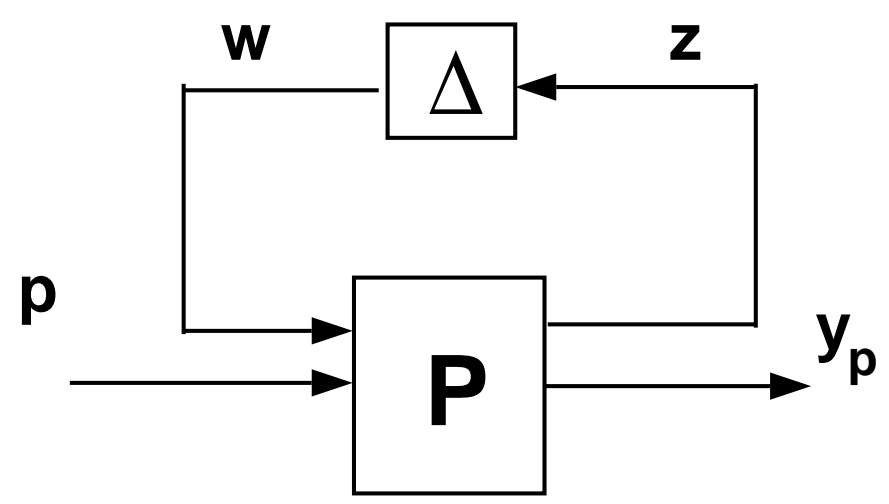

Fig. 3. Block Diagram of the Synthesized System

where $w=\Delta z$. The uncertainty block, $\Delta$ includes uncertainties from both components, i.e.,

$$
\Delta=\left[\begin{array}{cc}
\Delta_{1} & 0 \\
0 & \Delta_{2}
\end{array}\right]
$$

The uncertainties represented by $\Delta$ may include parametric and nonparametric uncertainties in each of the components. Moreover, the uncertainties can include structured or unstructured uncertainties in each component. Parametric uncertainties include uncertainties in modal frequencies, damping ratios, and mode shapes of the components. The nonparametric uncertainties considered here are the unmodeled dynamics of the components. Nonparametric uncertainties would typically be due to the truncated modes of the components.

If, as typically done, modal models of the components are used to describe their dynamics, then uncertainties in modal frequency and damping are embedded in the elements of the component state matrices $A_{1}$ and $A_{2}$. One can represent these uncertainties via blocks $\Delta A_{1}$ and $\Delta A_{2}$, such that the true state matrices are given by

$$
\begin{aligned}
& A_{1} \rightarrow A_{1}+\Delta A_{1} \\
& A_{2} \rightarrow A_{2}+\Delta A_{2}
\end{aligned}
$$

The parametric uncertainties given in Eqs. (47)-(48) may easily be put in a state space linear fractional representation form for robust control design and analysis [11], given by Eq. (45).

Consider the first component, and assume that there are $n_{1}$ distinct uncertainties associated with the $A_{1}$. Then, one may write

$$
A_{1} \rightarrow A_{1}+\sum_{i=1}^{n_{1}} Q_{i} \delta_{i}
$$

where matrix $Q_{i}$ represents the influence matrix for the $\delta_{i}$ uncertainty. Now, the influence matrices $E_{1}$ and $F_{1}$ (see Eqs. (10) and (12)) may be obtained from decomposition of matrices $Q_{i}, i=1, \ldots, n_{1}$. Factor each matrix as

$$
Q_{i}=E_{1 i} F_{1 i}
$$

where $E_{1 i} \in R^{n \times r_{i}}, F_{1 i} \in R^{r_{i} \times n}$, and $r_{i}$ denotes the rank of $Q_{i}$. Singular Value Decomposition (SVD) is a good way of performing this factorization. Then, the influence matrices $E_{1}$ and $F_{1}$ may be written as

$$
E_{1}=\left[\begin{array}{llll}
E_{11} & E_{12} & \ldots & E_{1 n_{1}}
\end{array}\right]
$$




$$
F_{1}=\left[\begin{array}{c}
F_{11} \\
F_{12} \\
\vdots \\
F_{1 n_{1}}
\end{array}\right]
$$

Matrices $E_{2}$ and $F_{2}$ of the second component may be obtained similarly. With matrices $E_{1}, E_{2}, F_{1}$, and $F_{2}$ defined, the synthesized system represented by Eq. (45) is in suitable form for robustness analysis or control design.

\subsection{Component Frequency and Damping Uncertainty at System Level}

In some applications, a model of the combined structure is available either from full-up structural analysis or system identification, i.e., no component synthesis is required to generate the model. However, the model uncertainty is defined at the component level, where substantially more effort is typically spent for modeling, testing, and verification. In order to perform robustness analysis or robust control design, the uncertainty formulation has to be redefined at the system level. Assume, without any loss of generality, that the overall model at the system level is in a real modal form. Then, the model of the system may be written as

$$
\left\{\begin{array}{c}
\dot{x}_{s} \\
y_{p} \\
z
\end{array}\right\}=\left[\begin{array}{ccc}
A_{s} & H_{s} & E_{s} \\
L_{s} & D_{s} & D_{E s} \\
F_{s} & 0 & 0
\end{array}\right]\left\{\begin{array}{c}
x_{s} \\
p \\
w
\end{array}\right\}
$$

Here, $A_{s}$ has the following form

$$
A_{s}=\left[\begin{array}{cccc}
A_{s_{1}} & 0 \ldots & 0 & \\
0 & \ddots & \ldots & 0 \\
\vdots & & \ddots & \vdots \\
0 & \ldots & 0 & A_{s_{m}}
\end{array}\right]
$$

with

$$
A_{s_{i}}=\left[\begin{array}{cc}
\lambda_{i_{R}} & -\lambda_{i_{I}} \\
\lambda_{i_{I}} & \lambda_{i_{R}}
\end{array}\right]
$$

where $\lambda_{i_{R}}$ and $\lambda_{i_{I}}$ denote the real and imaginary part of the ith eigenvalue pair of the system. Here, we assume that the eigenvalues are ordered from smallest to the largest. From component synthesis, we know that the first $m$ eigenvalues of matrix $\hat{A}_{\alpha \alpha}$ should closely match the eigenvalues of the $A_{s} . m$ is typically chosen to be smaller than the number of system modes that are within half the bandwidth of the component dynamics. Let matrix $\chi$ denote a real similarity transformation through which matrix $\hat{A}_{\alpha \alpha}$ is put in real modal form, with eigenvalue blocks in increasing order, and let $\Psi=\chi^{-1}$. Such a transformation may be obtained from the complex eigenvectors of $\hat{A}_{\alpha \alpha}$ by collecting the real and imaginary parts of the eigenvectors corresponding to complex conjugate pairs of eigenvalues. Applying this similarity transformation to the system in Eq. (45) gives

$$
\left\{\begin{array}{c}
\dot{\gamma} \\
y_{p} \\
z
\end{array}\right\}=\left[\begin{array}{ccc}
\Psi \hat{A}_{\alpha \alpha} \chi & \Psi \hat{H}_{\alpha} \Psi \hat{E}_{\alpha} \\
\hat{L}_{\alpha} \chi & \hat{D}_{\alpha} & D_{E \alpha} \\
\hat{F}_{\alpha} \chi & 0 & 0
\end{array}\right]\left\{\begin{array}{l}
\gamma \\
p \\
w
\end{array}\right\}
$$

Comparing the system in Eq. (56) with that given by Eq. (53), and keeping in mind that the first $m$ eigenvalues of the two systems should match, it is observed that the uncertainties at the component level can not be put exactly in the same structure 
at the system level, mainly due to the additional dynamics in the synthesized model that is neglected. However, a reasonable approximation may be made by choosing $E_{S}$ and $F_{s}$ as

$$
E_{s}=\Psi_{1} \hat{E}_{\alpha} ; F_{s}=\hat{F}_{\alpha} \chi_{1}
$$

where $\Psi_{1}$ and $\chi_{1}$ denote partitions formed from the first $2 \times m$ rows and $2 \times m$ columns of $\Psi$ and $\chi$, respectively. With $E_{s}$ and $F_{s}$ defined, the global system is prepared for robust design and analysis. It should be noted that if more than one rigid-body mode are present in the system (e.g., space applications), then it would be unlikely that the rigid-body mode in $\hat{A}_{\alpha \alpha}$ would have an exact corresponding mode in $A_{s}$. This is because the eigenvectors corresponding to rigid-body modes (with repeated eigenvalues at zero) are not unique. One way to handle this is to replace the partition in matrices $A_{s}, H_{s}$, and

$N_{s}$, corresponding to the rigid-body modes, with those of $\Psi \hat{A}_{\alpha \alpha} \chi, \Psi \hat{H}_{\alpha}, \Psi \hat{E}_{\alpha}$, and $\hat{L}_{\alpha} \chi$, respectively. This note also applies to repeated flexible modes, except in many of such cases that have unique eigenvectors.

\subsection{Uncertainty in Mode Shapes}

In this section, the effects of uncertainty in the component mode shapes are considered. For simplicity of presentation, it is assumed that the performance output does not have feedthrough terms $\left(D_{p_{1}}\right.$, and $D_{p_{2}}$ are omitted). Moreover, it is assumed that the component models do not have any uncertainties in LFT form $\left(E_{i}, F_{i}, \Gamma_{i}\right.$, and $D_{E_{i}}, i=1,2$ are neglected). With a modal component model, the parametric uncertainties in the mode shape data affect the elements of the input and output influence matrices, which include matrices $C_{i}, H_{i}$, and $L_{i}, i=1,2$. This means that there would be uncertainty associated with the elements of the basis vectors $N_{c}$, as seen from Eq. (26). Here we assume that the uncertainty in the mode shapes, whether structured or unstructured, can be translated into an uncertainty representation for $N_{c}$. With this assumption, the actual basis vectors $N_{c}$ may be represented in various representations of uncertainty, such as additive, multiplicative, etc. Let $N_{c}$ be represented as follows

$$
N_{c} \rightarrow N_{c}+R_{N} \Delta_{N}
$$

where $R_{N}$ is a chosen basis for uncertainty influence, and $\Delta_{N}$ represent the uncertainty, which can be structured or unstructured. The effects of mode shape uncertainty in the influence matrices $H_{i}$, and $L_{i}, i=1,2$, can be characterized in the same way, i.e.,

$$
\begin{gathered}
H \rightarrow H+R_{H} \Delta_{H} \\
L \rightarrow L+R_{L} \Delta_{L}
\end{gathered}
$$

Using Eqs. (58)-(60) in Eq. (37), and assuming that there are no uncertainties in the frequency and damping data or nonparametric uncertainties present, one obtains

$$
\begin{gathered}
\dot{\alpha}=\left(\hat{A}_{\alpha \alpha}+\Delta \hat{A}_{\alpha \alpha}\right) \alpha+\left(\hat{H}_{\alpha}+\Delta H_{\alpha}\right) p \\
y_{p}=\left(\hat{L}_{\alpha}+\Delta \hat{L}_{\alpha}\right) \alpha
\end{gathered}
$$

where

$$
\Delta \hat{A}_{\alpha \alpha}=\Delta_{N}{ }^{T} R_{N}^{T} A R_{N} \Delta_{N}+\Delta_{N}{ }^{T} R_{N}^{T} A N_{c}+N_{c}^{T} A R_{N} \Delta_{N}
$$




$$
\begin{gathered}
\Delta \hat{H}_{\alpha}=\Delta_{N}^{T} R_{N}^{T} R_{H} \Delta_{H}+\Delta_{N}^{T} R_{N}^{T} H+N_{c}^{T} R_{H} \Delta_{H} \\
\Delta \hat{L}_{\alpha}=R_{L} \Delta_{L} R_{N} \Delta_{N}+R_{L} \Delta_{L} N_{c}+L R_{N} \Delta_{N} \\
A=\left[\begin{array}{cc}
A_{1} & 0 \\
0 & A_{2}
\end{array}\right] ; H=\left[\begin{array}{cc}
H_{1} & 0 \\
0 & H_{2}
\end{array}\right] ; L=\left[\begin{array}{cc}
L_{1} & 0 \\
0 & L_{2}
\end{array}\right]
\end{gathered}
$$

From these equations, it is observed that the input and output parametric uncertainties in the dynamics of the components, in the original coordinates, diffuse into input, output, and state parametric uncertainties in the transformed coordinates representation of the synthesized system dynamics. Moreover, the state parametric uncertainties involve a quadratic form of the uncertainty $\Delta$, which can be presented efficiently as an LFT. [12,13]

\subsection{Unmodeled Dynamics}

The traditional approach in substructure synthesis has been to use higher bandwidth in the dynamics of the components to be synthesized, roughly twice the bandwidth of interest for the combined structure. This approach has worked successfully in many applications. However, it is somewhat ad hoc, with some potential shortcomings. First, it does not provide guaranteed levels of accuracy for the predicted system parameters, such as frequencies, damping ratios, etc. Second, it may suffer from the finite element method's potential loss of numerical accuracy for higher frequency modes in addition to a loss of correlation with measured frequencies. As mentioned earlier, control design requires accurate assessment of model parameters or potential uncertainties. Traditionally, unmodeled dynamics, which typically include the higher frequency modes not included in the design model, have been treated via additive uncertainties in the plant model, which forces the control system to roll off to avoid potentially destablizing spillover problems. Unfortunately, unmodeled (truncated) dynamics at the component level generally do not correspond to the same in the synthesized (combined) system. Consequently, it is imperative that the effects of unmodeled dynamics in the components be characterized at the system level for proper dynamics and controls design and analysis.

The approach taken here is similar to the treatment for parametric uncertainty, which essentially considered the effects of uncertainty in the transformed coordinates. Rewrite the dynamics of the components, including the unmodeled dynamics, in a first-order form.

$$
\begin{aligned}
& \text { component } i=1 \text { or } 2 \\
& \left\{\begin{array}{c}
\dot{x}_{i} \\
\dot{\bar{x}}_{i}
\end{array}\right\}=\left[\begin{array}{cc}
A_{i} & 0 \\
0 & \bar{A}_{i}
\end{array}\right]\left\{\begin{array}{l}
x_{i} \\
\bar{x}_{i}
\end{array}\right\}+\left[\begin{array}{c}
B_{i} \\
\bar{B}_{i}
\end{array}\right] u+\left[\begin{array}{c}
H_{i} \\
\bar{H}_{i}
\end{array}\right] p_{i} \\
& y_{i}=\left[C_{i} \bar{C}_{i}\right]\left\{\begin{array}{l}
x_{i} \\
\bar{x}_{i}
\end{array}\right\} \\
& y_{p_{i}}=\left[L_{i} \bar{L}_{i}\right]\left\{\begin{array}{l}
x_{i} \\
\bar{x}_{i}
\end{array}\right\}
\end{aligned}
$$

The overbars indicate terms associated with the unmodeled dynamics. It should be noted that in most applications an accurate knowledge of the parameters associated with the unmodeled dynamics is not available, thus they are typically characterized in the form of uncertainty. Also, note that, similar to the previous treatments, the feedthrough terms in the noninterface outputs have been omitted. Following the synthesis procedure outlined previously, the component dynamics, as given in Eqs. (67)-(69), are appended to obtain a system similar to the one given by Eqs. (22)-(25), except that the order of the states 
are rearranged such that the modeled dynamics' states appear first, followed by the unmodeled dynamics' states, i.e., the state vector is given by $\left[\begin{array}{llll}x_{1}^{T} & x_{2}^{T} & \bar{x}_{1}^{T} & \bar{x}_{2}^{T}\end{array}\right]^{T}$. Then, the space of feasible states (in the connectivity and compatibility sense) are characterized by an equation similar to Eq. (26), but for the expanded system, as follows

$$
\left[C_{1}-C_{2} \bar{C}_{1}-\bar{C}_{2}\right] N_{c}=0
$$

Following the coordinate transformation (similar to Eq. (27)) and deriving the interface vector $u$ to guarantee the compatibility of the components' displacements and rotations at the interface, results in the synthesized dynamics of the combined system, which in transformed coordinates takes the form

$$
\begin{gathered}
\dot{\alpha}=\hat{A}_{\alpha \alpha} \alpha+\hat{H}_{\alpha} p \\
y_{p}=\hat{L}_{\alpha} \alpha
\end{gathered}
$$

where

$$
\begin{gathered}
\hat{A}_{\alpha \alpha}=N_{c}^{T}\left[\begin{array}{cccc}
A_{1} & 0 & 0 & 0 \\
0 & A_{2} & 0 & 0 \\
0 & 0 & \bar{A}_{1} & 0 \\
0 & 0 & 0 & \bar{A}_{2}
\end{array}\right] N_{c} \\
\hat{H}_{\alpha}=N_{c}^{T}\left[\begin{array}{cc}
H_{1} & 0 \\
0 & H_{2} \\
\bar{H}_{1} & 0 \\
0 & \bar{H}_{2}
\end{array}\right] \\
\hat{L}_{\alpha}=\left[\begin{array}{cccc}
L_{1} & 0 & \bar{L}_{1} & 0 \\
0 & L_{2} & 0 & \bar{L}_{2}
\end{array}\right] N_{c}
\end{gathered}
$$

It is reasonable to expect that both $\left[C_{1}-C_{2}\right]$ and $\left[C_{1}-C_{2} \bar{C}_{1}-\bar{C}_{2}\right]$ are full rank. Define the null space of the matrix $\left[C_{1}-C_{2}\right]$ by the matrix $N_{c_{m}}$, whose columns are orthogonal. Then, it can easily be shown that the columns of matrix $\left[\begin{array}{c}N_{c_{m}} \\ 0\end{array}\right]$ are included in the null space of $\left[C_{1}-C_{2} \bar{C}_{1}-\bar{C}_{2}\right]$, represented by the matrix $N_{c}$, from Eq. (70). Now, choose and partition $N_{c}$, such that

$$
N_{c}=\left[\begin{array}{cc}
N_{c_{m}} & N_{\bar{c}_{m}} \\
0 & N_{\bar{c}_{u}}
\end{array}\right]
$$

Here, the first column partition corresponds to the states of the modeled dynamics, and the second partition to the states of the unmodeled dynamics. Moreover, the first partition corresponds to the null space of matrix $\left[C_{1}-C_{2}\right]$. Using Eq. (76) in Eqs. (71) and (72), and separating the states, gives

$$
\dot{\alpha}_{m}=\tilde{A}_{m m} \alpha_{m}+\tilde{A}_{m u} \alpha_{u}+\tilde{H}_{m} p
$$




$$
\dot{\alpha}_{u}=\tilde{A}_{u m} \alpha_{m}+\tilde{A}_{u u} \alpha_{u}+\tilde{H}_{u} p
$$

with

$$
y_{p}=\tilde{L}_{m} \alpha_{m}+\tilde{L}_{u} \alpha_{u}
$$

where

$$
\begin{gathered}
{\left[\begin{array}{cc}
\tilde{A}_{m m} & \tilde{A}_{m u} \\
\tilde{A}_{u m} & \tilde{A}_{u u}
\end{array}\right]=\left[\begin{array}{cc}
N_{c_{m}} & N_{\bar{c}_{m}} \\
0 & N_{\bar{c}_{u}}
\end{array}\right]^{T}\left[\begin{array}{cccc}
A_{1} & 0 & 0 & 0 \\
0 & A_{2} & 0 & 0 \\
0 & 0 & \bar{A}_{1} & 0 \\
0 & 0 & 0 & \bar{A}_{2}
\end{array}\right]\left[\begin{array}{cc}
N_{c_{m}} & N_{\bar{c}_{m}} \\
0 & N_{\bar{c}_{u}}
\end{array}\right]} \\
{\left[\begin{array}{c}
\tilde{H}_{m} \\
\tilde{H}_{u}
\end{array}\right]=\left[\begin{array}{cc}
N_{c_{m}} & N_{\bar{c}_{m}} \\
0 & N_{\bar{c}_{u}}
\end{array}\right]^{T}\left[\begin{array}{cc}
H_{1} & 0 \\
0 & H_{2} \\
\bar{H}_{1} & 0 \\
0 & \bar{H}_{2}
\end{array}\right]} \\
{\left[\tilde{N}_{m} \tilde{N}_{u}\right]=\left[\begin{array}{cccc}
L_{1} & 0 & \bar{L}_{1} & 0 \\
0 & L_{2} & 0 & L_{2}
\end{array}\right]\left[\begin{array}{cc}
N_{c_{m}} & N_{\bar{c}_{m}} \\
0 & N_{\bar{c}_{u}}
\end{array}\right]}
\end{gathered}
$$

Note from Eq. (77) that $\tilde{A}_{m m}$ is the same as $\hat{A}_{\alpha \alpha}$ of the reduced dynamics for the baseline combined model (see Eq. (37)), so that if no unmodeled dynamics were present, Eq. (77) would represent the synthesized structure. Furthermore, the state equations for $\alpha_{m}$ and $\alpha_{u}$ are coupled. One way of looking at this is to imagine that there are two systems, one attributed to the modeled dynamics ( $\alpha_{m}$ states) and the other to the unmodeled dynamics $\left(\alpha_{u}\right.$ states), which are in feedback connection. In other words, the uncertainties associated with unmodeled dynamics in the components, which are typically represented by additive uncertainty to the component models, takes the form of an uncertainty in a feedback loop with the nominal combined structure, when the synthesized dynamics are considered.

\section{Numerical Results}

The proposed approach for synthesis and control design of flexible systems with component-level uncertainties is applied to a two-component planar problem shown in Figure 4. Here, a docking scenario involving two flexible space systems is considered. The two systems are modeled as planar free-free Euler-Bernoulli beams. The material and geometric properties of the system were chosen to provide considerable modal content in the low-mid frequency range to make the synthesis and control design task more challenging, and are provided in Table 1. It is assumed that in the docked configuration component no. 1 would provide attitude and position control for the combined system.

Table 1. Geometric and Structural Properties

\begin{tabular}{cccc} 
Property & Comp. 1 & Comp. 2 & Whole \\
\hline Length & 15 & 5 & 20 \\
Mass/Length & 6.775 & 6.775 & 6.775 \\
Rigidity, EI & 36458.3 & 36458.3 & 36458.3 \\
No. of modes & 20 & 20 & 20 \\
\hline
\end{tabular}




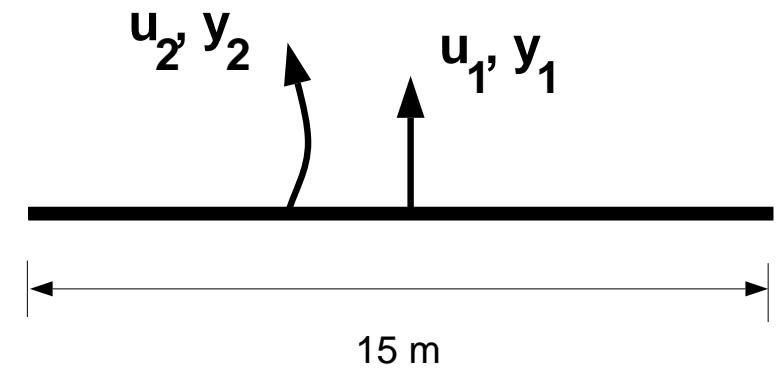

Component No. 1

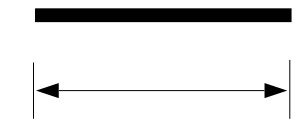

$5 \mathrm{~m}$

Fig. 4. Components of the Free-Free Problem

\subsection{Control Design}

The control design for component no. 1 is briefly described. As shown in Fig. 4, there are two control inputs available for this component. There is a torque wheel at the third of the length $(x=5 \mathrm{~m})$ and there is a thruster at the middle of the beam $(\mathrm{x}=7.5 \mathrm{~m})$ to provide attitude and position control. Collocated with these actuators are attitude and position sensors, providing measurement for the feedback loop. Here, sensor and actuator dynamics are not considered. The control design was based on the $H_{\infty}$ synthesis. [11,14] The performance requirements were defined in terms of the weighted output sensitivity transfer function from disturbances to the measurement sensors with assumed unstructured plant model uncertainty. Weighting functions were assigned to the performance outputs, as well as the inputs from the uncertainty block. The weighting function for the performance output was chosen as $W_{p}=\frac{0.002(s+100)}{(s+0.0001)(s+10)}$ for each channel to provide good disturbance rejection at low to mid frequencies, as well as to provide integral action to minimize steady-state errors. An unstructured, inputmultiplicative, form was used to represent the uncertainty in the system model. The weighting function, used to scale or distribute the uncertainty, was chosen as $W_{u}=\frac{(s+50)}{(s+500)}$ to emphasize model uncertainty in the mid-high frequency range. A 10th-order model of the beam, which included the first three flexible modes, was used in the control design. The controller was synthesized using the "hinfsyn" routine of the $\mu$-Analysis and Synthesis Toolbox [14]. The $H_{\infty}$ controller is designed in some sense to minimize the $H_{\infty}$ norm of the transfer function from disturbances to the performance outputs, while ensuring the closed-loop system remains stable for all allowed uncertainties. The $H_{\infty}$ design converged at a $\gamma$ value of 0.942 (note a $\gamma<1$ indicates that all the design requirements are met), and resulted in a 16th-order controller. Figure 5 provides a Bode plot of the controller. This controller was later used in robustness analysis of the system in the docked configuration.

\subsection{Robustness Analysis}

The two components were assumed to be in a docked configuration. Each of the components was modeled using the first 20 modes (including two rigid-body modes and 18 flexible modes), resulting in a 40th-order state space model for each of the substructures. The 20 modes used were deemed sufficient to provide a basis for describing the first 20 modes of the combined structure. The combined structure was also modeled globally (without synthesis) as a 20-m long beam using its first 20 modes, resulting in a 40th-order state space model. This model was used for validation of the synthesis approach and is referred to as the truth model. Using the procedure outlined in Eqs. (37)-(39), the dynamics of the two components were synthesized. Table 2 compares the natural frequencies of the synthesized model vs. those of the validation (truth) model. Comparison of the frequencies indicates a good match between the two models. 

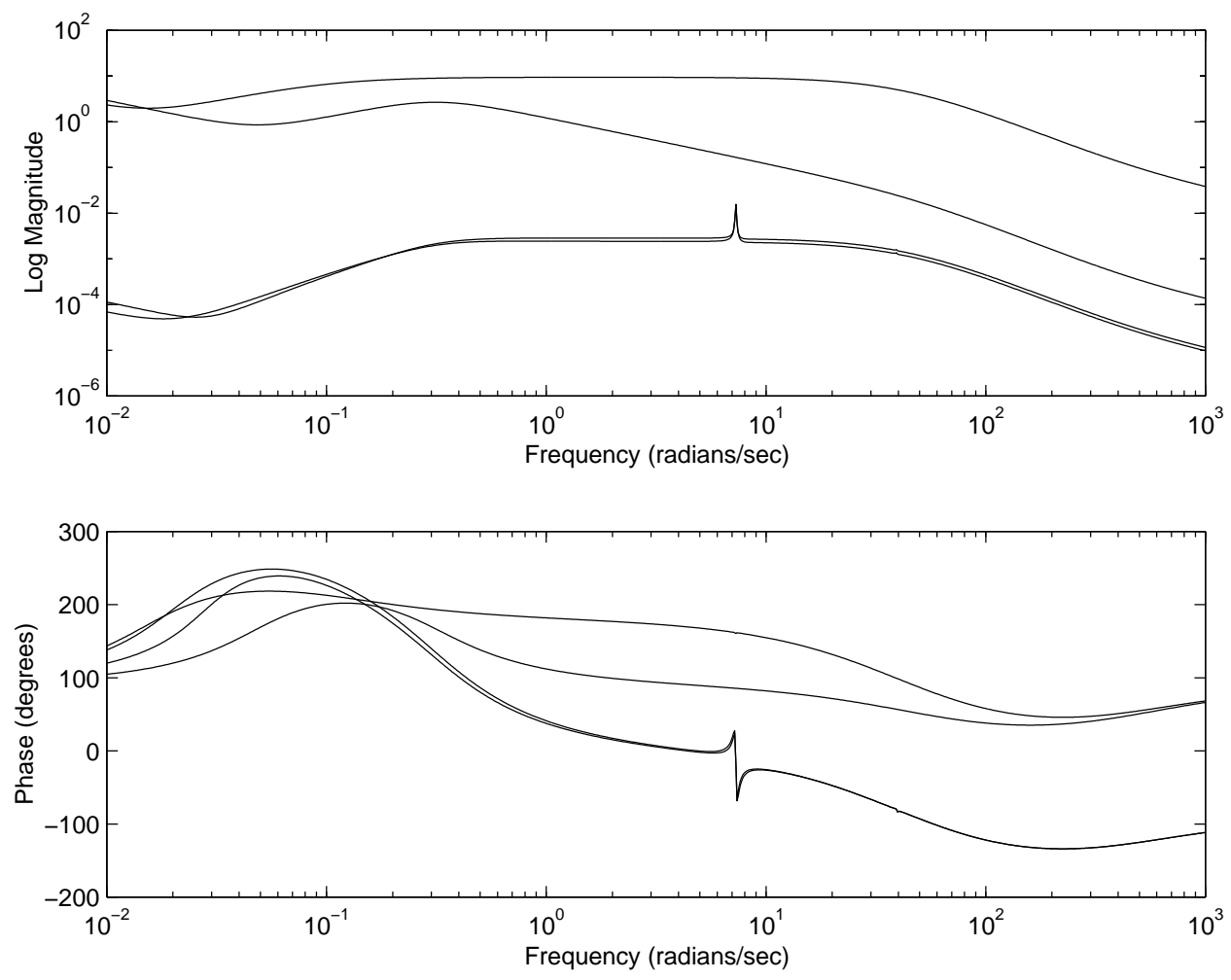

Fig. 5. Bode Plot for the $H_{\infty}$ Controller for Component No. 1

Table 2. Natural Frequencies

Mode No. True Synthesized

\begin{tabular}{ccc} 
Mode No. & True & Synthesized \\
\hline 1 & 0 & 0 \\
2 & 0 & 0 \\
3 & 0.0410 & 0.0414 \\
4 & 0.1131 & 0.1158 \\
5 & 0.2217 & 0.2264 \\
6 & 0.3665 & 0.3676 \\
7 & 0.5475 & 0.5500 \\
8 & 0.7647 & 0.7809 \\
9 & 1.0181 & 1.0389 \\
10 & 1.3077 & 1.3108 \\
11 & 1.6335 & 1.6437 \\
12 & 1.9955 & 2.0425 \\
13 & 2.3938 & 2.4466 \\
14 & 2.8282 & 2.8344 \\
15 & 3.2988 & 3.3251 \\
16 & 3.8056 & 3.9045 \\
17 & 4.3488 & 4.4533 \\
18 & 4.9278 & 4.9389 \\
19 & 5.5432 & 5.6002 \\
20 & 6.1948 & 6.3764 \\
\hline
\end{tabular}

Maghami, 16 


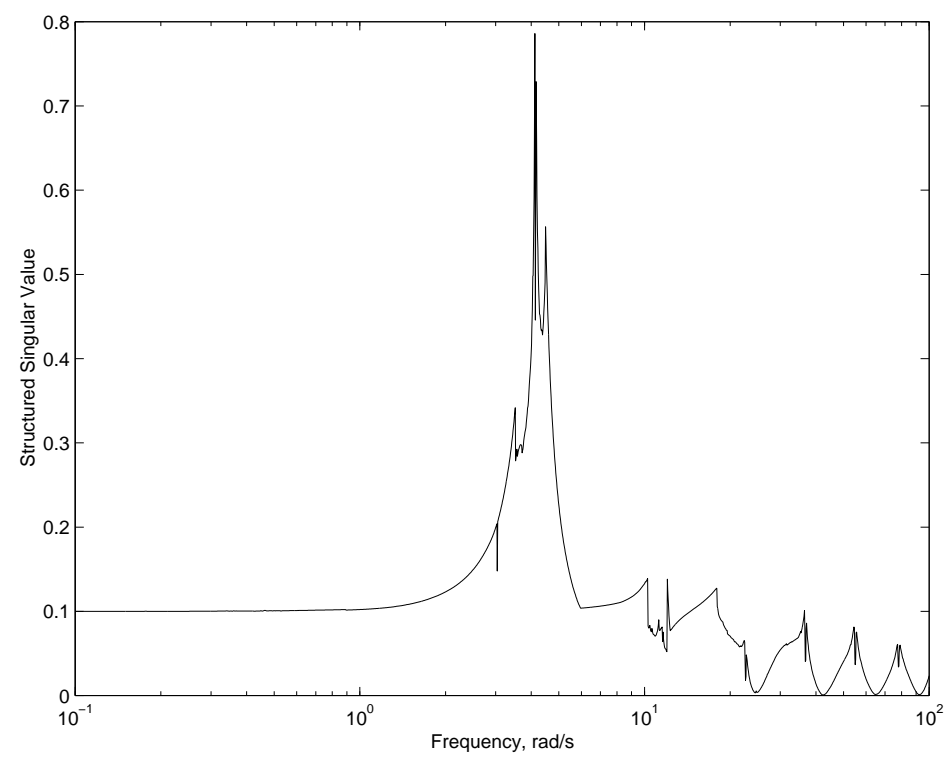

Fig. 6. Robust Stability $\mu$ Plot for the Synthesized System

Now, both the synthesized model as well as the truth model were assessed against uncertainties in the frequencies and damping of the two components. Specifically, it was assumed that there is a five percent uncertainty associated with the frequency of the first mode in each component. Furthermore, it was assumed that there is a 15 percent uncertainty associated with the damping of the first mode in each component. The inherent structural damping ratio used for each of the component was chosen at 0.5 percent. The damping ratio used for the truth model was based on the values obtained from the synthesized model.

First, robust stability of the synthesized model was assessed. Following Eq. (49), the coefficients $Q_{i}$ for the four uncertainty terms in the state matrices of the components, corresponding to frequency and damping in their first mode, were defined and factored according to Eq. (50). Note that the uncertainties were assumed to be distinct, real scalars. Using the system defined in Eq. (45) (with appropriate input/output matrices for the uncertainties) together with the $H_{\infty}$ controller designed for component no. 1, a robust stability analysis was performed with aid of the "mu" routine of $\mu$-Analysis and Synthesis Toolbox [14]. A Mu plot of the closed-loop system is provided in Fig. 6, where it is observed that the structured singular value $\mu$ is less than one for all frequencies. Therefore, the synthesized system with the $H_{\infty}$ controller is robustly stable.

Next, robust stability of the truth model against the same uncertainties in the component models was assessed. The first task was to approximately translate the uncertainties from the component level to the system level. This was performed by transforming the state matrix of the synthesized system to real modal form, with eigenvalues in increasing order on the diagonal. Then, Eq. (57) was used to determine the influence matrices at the system level, and the truth model given by Eq. (52) was used in robust stability analysis. Again, the $H_{\infty}$ controller designed for component no. 1 was the controller used in the robustness analysis of the closed-loop truth model. A Mu plot of the closed-loop system is provided in Fig. 7 , where it is observed that the structured singular value $\mu$ is less than one for all frequencies. Comparing Figs. 6 and 7, it is observed the Mu plots for the synthesized and truth systems match each other reasonably well. This validates the approximate characterization of the uncertainty at the system level from component level definitions.

\section{Concluding Remarks}

A novel method for synthesizing the dynamics of substructures has been developed which views the interface forces and moments as input vectors. The synthesized structure can be realized in certain transformed coordinates, which has the advantage of being more amenable to uncertainty and robustness analysis and design, as well as being smaller in order. An approach for formulating robustness of flexible systems in the presence of frequency and damping uncertainties at the component level was presented. The effects of component mode shape uncertainties as well as unmodeled component dynamics on the dynamics and robustness of the synthesized system were investigated, and characterized in transformed coordinates. The robustness of non-synthesized dynamic models with component-level uncertainties was considered by approximately 


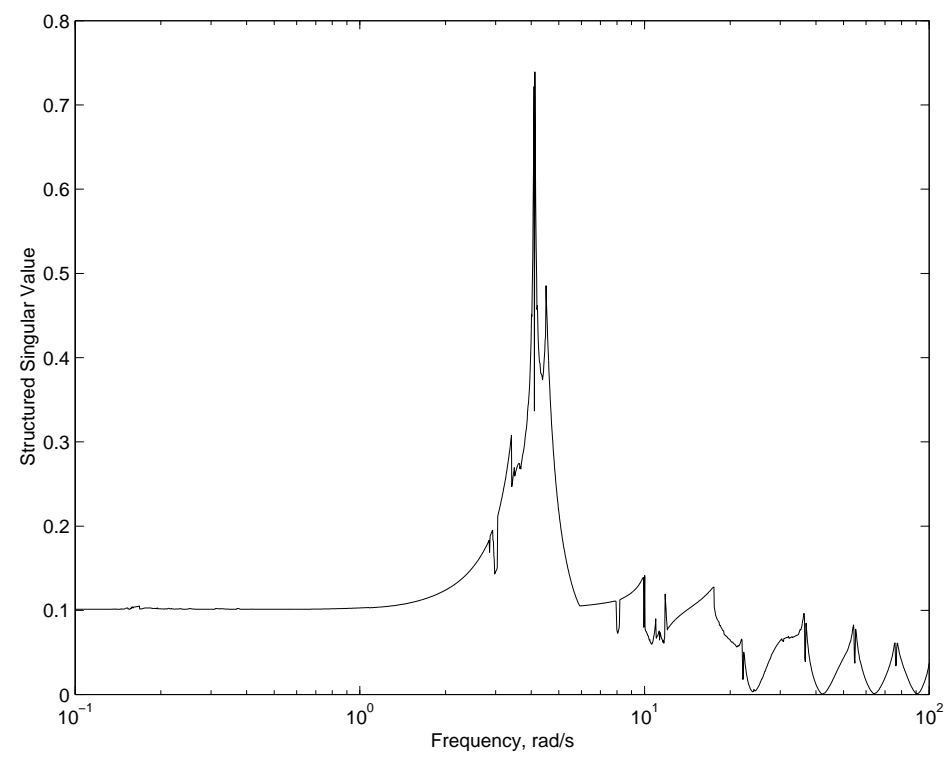

Fig. 7. Robust Stability $\mu$ Plot for the Truth System

projecting uncertainties from component levels to system level. A numerical example involving a two-dimensional simulated docking problem demonstrated the feasibility of the proposed approach.

\section{References}

[1] Hurty, W., "Vibrations of Structural systems by Component Mode Synthesis," Journal of Engineering Mechanics Division, Proc. of ASCE, 1960, pp. 51-69.

[2] Craig, R., Jr. and Bampton, M., "Coupling of Substructures for Dynamic Analysis," AIAA Journal, Vol. 6, No. 7, 1968, pp. 1313-9.

[3] Hintz, R., "Analytical Methods in Component Mode Synthesis," AIAA Journal, Vol. 13, No. 8, 1975, pp. 1007-16.

[4] Craig, R., Jr., "Methods of Component Mode Synthesis," Shock and Vibration Digest, Vol. 9, No. 11, 1977, pp. 3-10.

[5] Craig, R., Jr., Structural Dynamics: Introduction to Computer Methods, Wiley, 1981.

[6] Meirovitch, L. and Hale, A., "On the Substructure Synthesis Method," AIAA Journal, Vol. 19, No. 7, 1981, pp. 940-47.

[7] Kana, D. and Huzar, S., "Synthesis of Shuttle Vehicle Damping using Substructure Test Results," AIAA Journal of Spacecraft and Rocket, Vol. 10, Dec. 1973, pp. 790-7.

[8] Su, T.-J., Babuska, V., and Craig, R. R., Jr., "Substructure-Based Controller Design Method for Flexible Structures," Journal of Guidance, Control, and Dynamics, Vol. 18, No. 5, 1995, pp. 10531061.

[9] Lim, K., "Robust Control Design Framework for Substructure Models," AIAA, Journal of Guidance, Control, Dynamics, Vol. 19, No. 1, Jan.-Feb. 1996, pp. 181-190.

[10] Sunar, M. and Rao, S. S., "Optimal Structural Control by Substructure Synthesis," AIAA Journal, Vol. 38, No. 11, November 2000, pp. 2191-2194.

[11] Zhou, K., "Robust and Optimal Control," Prentice-Hall, Upper Saddle River, NJ, 1996.

[12] Belcastro, C., "Parametric Uncertainty Modeling: An Overview," Proc. of American Control Conference, Philadelphia, PA, 1998, pp. 992-6.

[13] Belcastro, C., "On the Numerical Formulation of Parametric Linear Fractional Transformation Uncertainty Models for Multivariate Matrix Polynomial Problems,” Tm-1998-206939, NASA, Nov. 1998.

[14] Balas, G. J., Doyle, J. C., Glover, K., Packard, A., and Smith, R., $\mu$-Analysis and Synthesis Toolbox, The MathWorks, Inc., 1994-2008. 